\title{
Analysis of Long Noncoding RNA Expression Profile in Human Pulmonary Microvascular Endothelial Cells Exposed to Lipopolysaccharide
}

\author{
Dong Wang Changping Gu Mengjie Liu Ge Liu Huan Liu \\ Yuelan Wang \\ Department of Anesthesiology, Qianfoshan Hospital, Shandong University, Jinan, China
}

\section{Key Words}

Long noncoding RNA Expression profile $\cdot$ Acute lung injury $\cdot$ Lipopolysaccharide $\cdot$ Human pulmonary microvascular endothelial cell

\begin{abstract}
Background/Aims: Acute lung injury (ALI) and acute respiratory distress syndrome (ARDS) are a continuum of life-threatening lung changes. Pulmonary vascular injury is one of the most important initial causes of ALI and ARDS. However, the functions of long noncoding RNAs (IncRNAs) in pulmonary endothelial injury remain largely unknown. The aim of the present study was to determine the IncRNA expression profile of human pulmonary microvascular endothelial cells (HPMECs) exposed to lipopolysaccharide (LPS) and explore the potential functions of differentially expressed IncRNAs. Methods: Microarray analysis was used to identify differentially expressed IncRNAs and mRNAs. Bioinformatics analyses, including Gene Ontology (GO), Kyoto Encyclopedia of Genes and Genomes (KEGG) pathway, IncRNA-mRNA coexpression network and transcription factor (TF)-IncRNA network analyses, were performed to predict the functions of significantly differentially expressed IncRNAs and mRNAs. Realtime polymerase chain reaction (PCR) was used to determine the expression of selected IncRNAs and mRNAs. Results: In this study, we found that 213 IncRNAs and 212 mRNAs were significantly differentially expressed in HPMECs exposed to LPS (fold change $>2.0, p<0.05$ ). Furthermore, we found that mRNAs co-expressed with IncRNAs were significantly enriched in the TNF signaling pathway, the NF-KB signaling pathway, cell adhesion molecules (CAMs), cytokine-cytokine receptor interactions, and extracellular matrix (ECM)-receptor interactions. The expression levels of all but one of the selected IncRNAs and mRNAs detected by real-time PCR were similar to those detected by microarray analysis. Conclusion: Our data indicate that IncRNAs play an important role in LPS-induced pulmonary endothelial inflammation and barrier dysfunction and may be potential preventive and therapeutic targets for ALI and ARDS.




\section{Cellular Physiology Cell Physiol Biochem 2019;52:653-667

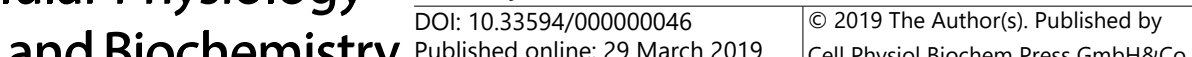 \\ \begin{tabular}{ll} 
Published online: 29 March 2019 Cell Physiol Biochem Press GmbH\&Co. KG \\
\hline Wh
\end{tabular} \\ Wang et al.: IncRNA in HPMECs Exposed to LPS}

\section{Introduction}

Acute lung injury (ALI) and acute respiratory distress syndrome (ARDS) are part of a continuum of life-threatening lung changes that interfere with the diffusion of oxygen from alveoli into the blood [1-4]. Sepsis and pneumonia are the most common causes of ARDS, and pulmonary vascular injury is one of the most important initial causes of ALI and ARDS. ARDS accounts for $10 \%$ of intensive care unit admissions globally. Despite years of basic and clinical studies, the global mortality of ARDS has been as high as $40 \%$ in recent years $[5,6]$. For these reasons, the identification of key molecules involved in ARDS is highly demanded for improving the clinical outcome of this syndrome.

It is now generally accepted that the majority of mammalian RNA transcripts are noncoding RNAs (ncRNAs). Long noncoding RNAs (lncRNAs), which are longer than 200 nucleotides, are generally not translated into proteins [7-10]. By regulating gene expression at posttranscriptional, transcriptional and epigenetic stages, IncRNAs participate in most essential biological processes. Over the past decade, the study of lncRNAs has become a hot spot in basic and clinical research of ALI and ARDS. Recent studies have shown that miRNAs are involved in the pathophysiology of ARDS and may be interesting diagnostic biomarkers and therapeutic targets [11-20]. However, less is known about the role of lncRNAs in the pathogenesis of ALI and ARDS.

To study the function of IncRNAs in the pathogenesis of ALI and ARDS, we established an experimental model of pulmonary endothelial inflammation and barrier dysfunction by stimulating HPMECs with LPS [21-24] and investigated the expression profile of IncRNAs and mRNAs by microarray analysis. We found that 213 lncRNAs and 212 mRNAs were significantly differentially expressed. Bioinformatics analyses indicated that the differentially expressed lncRNAs might play an important role in LPS-induced pulmonary endothelial inflammation and barrier dysfunction.

These findings will serve to increase the understanding of the pathogenesis of pulmonary endothelial dysfunction. Moreover, due to opportunities to identify novel therapeutic and preventive targets, our results may provide relevant information for future clinical interventions of ALI and ARDS.

\section{Materials and Methods}

\section{Cell culture, LPS treatment, and RNA isolation}

Human pulmonary microvascular endothelial cells (HPMECs, ScienCell, San Diego, CA, USA) were cultured in endothelial cell medium (ECM, ScienCell, San Diego, CA, USA) in a humidified 5\% $\mathrm{CO}_{2}$ incubator at $37^{\circ} \mathrm{C}$. LPS (Sigma, St Louis, MO, USA) from Escherichia coli 0111:B4 was dissolved in sterile water and prepared fresh at the time of use. At approximately $90 \%$ confluence in culture, HPMECs were starved for 1 hour $(\mathrm{h})$ in serum-free medium and then stimulated for $4 \mathrm{~h}$ with LPS $(1 \mu \mathrm{g} / \mathrm{ml})$ or vehicle control (PBS) in ECM containing 1\% FBS as previously described [14] [21] [25]. HPMECs were used in passage numbers 4 to 6. Total RNA was isolated using TRIzol reagent (Invitrogen, Carlsbad, CA, USA). The quality of the RNA preparations was verified on an Agilent 2100 bioanalyzer (Agilent Technologies, Santa Clara, CA, USA).

\section{Microarray analysis}

Total RNA was hybridized to Affymetrix Human Transcriptome Array 2.0 (Affymetrix, Santa Clara, CA, USA). Hybridized data were preprocessed and statistically analyzed as described previously [26, 27]. Differentially expressed lncRNAs and mRNAs were identified by fold-change screening at a threshold of 2.0fold or greater and a $p$ value $<0.05$. 
GO and KEGG pathway analyses

GO analysis (http://www. geneontology.org) was performed to explore functions of genes based on the biological pathway, cellular component and molecular function categories; Kyoto Encyclopedia of Genes and Genomes (KEGG) (http://www.genome.jp/kegg/) analysis was performed to determine pathways significantly enriched in genes. $P<0.05$ and FDR $<0.05$ were used as thresholds to define significantly enriched GO terms or pathways.
Table 1. Primers designed for real-time PCR of candidate lncRNAs and mRNAs. Tm: temperature. bp: base pair

\begin{tabular}{|c|c|c|c|c|}
\hline Primer name & Primer FW $\left(5^{\prime}-3^{\prime}\right)$ & Primer RW $\left(5^{\prime}-3^{\prime}\right)$ & $\mathrm{Tm}$ & Product length \\
\hline n384765 & AGATGGAAAGGGGTGCTTGG & GCAGAGTGTGAGAAGGGTGG & 60 & 119 \\
\hline n344917 & ACTCCAAGGCGTTTTTCCAC & GGAAGCCTCGACCCTGTATTG & 60 & 104 \\
\hline XLOC_12_015215 & GCTCATCACACAGAACTTTTCTCA & AGAACAAAATACTCTGGGGAGAAG & 60 & 103 \\
\hline n340107 & GTCCTCCTCATCTTCCTCTTCC & ACAGGCTCATCAGTTAGCATCT & 60 & 100 \\
\hline $\mathrm{n} 407205$ & GAGGAACACTGGGTTGGACT & TGCCACCCTGGGGAATATAAAG & 60 & 117 \\
\hline NR_001564 & АТTTCTTACTCTCTCGGGGCTG & CCATAAAGGGTGTTGGGGGA & 60 & 100 \\
\hline SELE & AGCCTTCAGTGTACCTCATCT & GCACCTCACAGAGCCATTC & 60 & 105 \\
\hline IL8 & TTCTAGGACAAGAGCCAGGAAG & ATCAGGAAGGCTGCCAAGAG & 60 & 102 \\
\hline VCAM1 & ATACCCTCCCAGGCACACA & CTCCAAGGATCACGACCATCT & 60 & 111 \\
\hline ICAM1 & ATGCCCAGACATCTGTGTCC & GGGGTCTCTATGCCCAACAA & 60 & 112 \\
\hline CXCL10 & ATATGGCACACTAGCCCCAC & GATTCATGGTGCTGAGACTGGA & 60 & 100 \\
\hline MMP10 & TTACATTGCTAGGCGAGATAGG & CAGTCACAGAACATGCAGGAA & 60 & 120 \\
\hline АВI3BP & GGCAGCAATGTATCACCAAAC & GAGCAGGTCGCACAACTATC & 60 & 109 \\
\hline TRPC6 & AGGATGACGCTGATGTGGAG & GGACTCGGCACCAGATTGA & 60 & 106 \\
\hline
\end{tabular}

\section{Real-time PCR}

A FastQuant RT Kit (Tiangen, Beijing, China) was used to reverse transcribe total RNA into cDNA following the manufacturer's directions. Real-time PCR was performed using SuperReal PreMix Plus (SYBR Green) (Tiangen, Beijing, China) in the Applied Biosystems GeneAmp® PCR System 9700. The reaction conditions were as follows: incubation at $95^{\circ} \mathrm{C}$ for $15 \mathrm{~min}$, followed by 40 cycles of $95^{\circ} \mathrm{C}$ for $10 \mathrm{~s}$ and $60^{\circ} \mathrm{C}$ for $20 \mathrm{~s}$. The relative expression levels of $\operatorname{lncRNAs}$ were calculated using the $2^{-\Delta \Delta \mathrm{Ct}}$ method and normalized to GAPDH levels [28]. The primers for each lncRNA and mRNA are listed in Table 1.

\section{LncRNA-mRNA coexpression network}

A IncRNA-mRNA coexpression network was constructed to identify the interactions between lncRNA and mRNA according to the normalized signal intensity of specific mRNA and IncRNA expression levels as described previously $[29,30]$.

\section{TF-IncRNA network}

Transcription factors (TFs) and DNA sequence motifs from 2.0 kilobase upstream of the transcription start site of differentially expressed lncRNAs were predicted with the TRANSFACT professional database (http://gene-regulation.com/). TFs with a matrix score and core score equal to 1 were selected.

\section{Statistical analysis}

All data are expressed as the mean \pm SEM. For comparisons between 2 groups, unpaired Student's t-test for parametric data and Mann-Whitney's U-test for nonparametric data were used. All statistical analyses were performed with GraphPad Prism 7.04 (GraphPad Software, San Diego, CA, USA). A $p$ value $<$ 0.05 was considered statistically significant.

\section{Results}

\section{Profiles of the differentially expressed IncRNAs and mRNAs}

To evaluate the differential expression of IncRNAs in HPMECs stimulated with LPS, we performed microarray analysis of the lncRNA expression profile using Affymetrix Human Transcriptome Array 2.0, which covers 245, 349 coding transcripts and 40, 914 noncoding transcripts.

We found that 213 IncRNAs were significantly differentially expressed (fold change $>$ $2.0, p<0.05)$. Of these, 189 lncRNAs were upregulated and 28 lncRNAs were downregulated (Fig. 1A, Fig. 1C). The top 20 most significantly upregulated (Table 2) and downregulated (Table 3) lncRNAs are listed below. At the same time, 212 mRNAs were significantly differentially expressed (fold change $>2.0, p<0.05$ ), including 183 upregulated mRNAs 


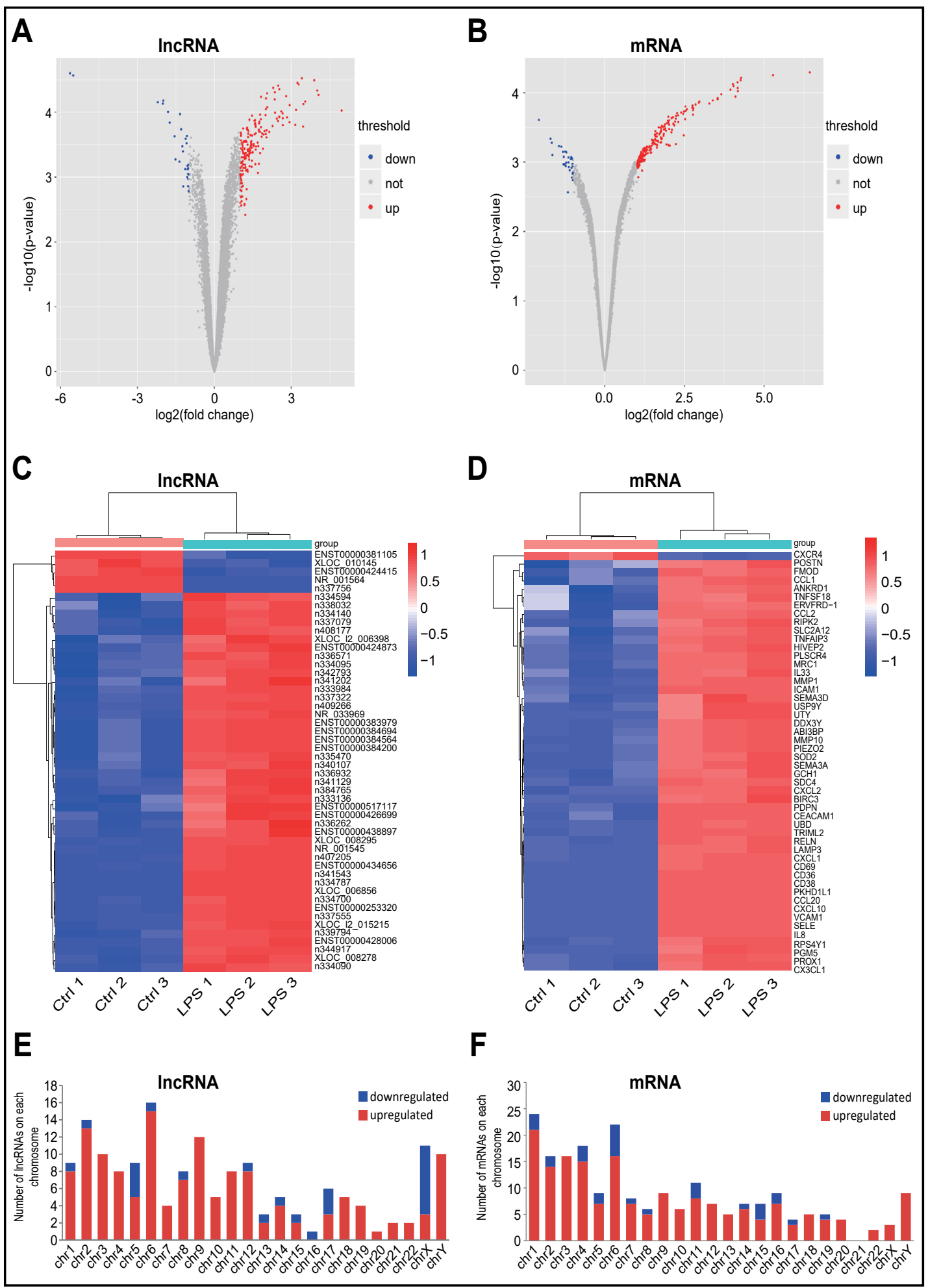

Fig. 1. Volcano plots, expression profiles and chromosome distribution of differentially expressed lncRNAs and mRNAs in HPMECs treated with LPS. Volcano plots of differentially expressed IncRNAs (A) and mRNAs (B). Gray dots indicate no change. Blue and red dots indicate significantly downregulated and upregulated lncRNAs and mRNAs, respectively. Hierarchical clustering indicates lncRNA (C) and mRNA (D) profiles. Red and blue columns refer to high and low relative expression, respectively. Distribution of differentially expressed lncRNAs (E) and mRNAs (F). 


\begin{tabular}{|c|c|c|}
\hline \multirow{2}{*}{$\begin{array}{l}\text { Cellular Physiology } \\
\text { and Biochemistry }\end{array}$} & \multicolumn{2}{|c|}{ Cell Physiol Biochem 2019;52:653-667 } \\
\hline & $\begin{array}{l}\text { DOl: 10.33594//000000046 } \\
\text { Published online: } 29 \text { March } 2019\end{array}$ & $\begin{array}{l}\text { O } 2019 \text { The Author(s). Published by } \\
\text { Cell Physiol Biochem Press GmbH\&Co. KG }\end{array}$ \\
\hline
\end{tabular}

Table 2. Top 20 significantly upregulated IncRNAs in HPMECs treated with LPS. Fold: fold change. Chr: chromosome number. LPS 1 to 3 and Ctrl 1 to 3: normalized gene signals of each sample

\begin{tabular}{lccccccccccc}
\hline Accession & P value & Fold & Chr & Gene start & Gene end & LPS1 & LPS2 & LPS3 & Ctrl & Ctrl & Ctrl \\
\hline n409266 & 0.00003 & 10.6 & chr3 & 14591012 & 14596896 & 10.4 & 10.5 & 10.6 & 7.30 & 7.00 & 7.20 \\
n337322 & 0.00003 & 14.8 & chr1 & 10266777 & 10266807 & 11.3 & 11.3 & 11.3 & 7.65 & 7.14 & 7.51 \\
XLOC_008278 & 0.00003 & 9.43 & chrY & 3904538 & 3968361 & 8.13 & 8.25 & 8.07 & 4.67 & 5.05 & 5.02 \\
n334140 & 0.00003 & 9.76 & chr1 & 43013360 & 43025500 & 10.0 & 9.86 & 10.0 & 6.43 & 6.92 & 6.65 \\
XLOC_12_015215 & 0.00004 & 4.89 & chr9 & 68773916 & 68778906 & 7.14 & 7.28 & 7.36 & 5.00 & 4.91 & 5.00 \\
NR_001545 & 0.00004 & 5.77 & chrY & 14774265 & 14804162 & 5.30 & 5.36 & 5.56 & 2.79 & 2.96 & 2.88 \\
n334090 & 0.00004 & 5.13 & chr3 & 13923725 & 13925844 & 8.67 & 8.56 & 8.47 & 6.11 & 6.17 & 6.35 \\
n344917 & 0.00005 & 4.16 & chr6 & 86386800 & 86388112 & 5.80 & 5.92 & 5.95 & 3.78 & 3.81 & 3.92 \\
XLOC_006856 & 0.00005 & 16.6 & chr8 & 79854913 & 79855506 & 7.06 & 7.92 & 7.67 & 3.62 & 3.45 & 3.41 \\
n407205 & 0.00005 & 6.95 & chr1 & 9906735 & 9913497 & 5.68 & 5.78 & 5.96 & 3.04 & 2.81 & 3.19 \\
ENST000004359 & 0.00005 & 3.52 & chr6 & 74779167 & 75400443 & 5.12 & 5.09 & 5.08 & 3.35 & 3.30 & 3.20 \\
n339794 & 0.00006 & 4.03 & chr1 & 10731474 & 10773583 & 7.76 & 7.76 & 7.84 & 5.73 & 5.67 & 5.93 \\
n408177 & 0.00006 & 5.09 & chrY & 16636454 & 16955848 & 6.78 & 6.60 & 6.53 & 4.23 & 4.48 & 4.16 \\
ENST000004280 & 0.00006 & 11.6 & chr9 & 13446525 & 13487510 & 7.47 & 7.41 & 7.81 & 3.84 & 3.74 & 4.48 \\
n334095 & 0.00007 & 11.0 & chr1 & 10396161 & 10396374 & 10.7 & 10.8 & 11.0 & 7.64 & 6.91 & 7.63 \\
ENST000004266 & 0.00007 & 7.63 & chrY & 3904538 & 3968361 & 7.07 & 7.63 & 7.55 & 4.29 & 4.70 & 4.47 \\
ENST000003845 & 0.00008 & 4.17 & chr1 & 15244554 & 15244653 & 5.69 & 5.73 & 5.72 & 3.86 & 3.50 & 3.60 \\
ENST000005179 & 0.00008 & 4.02 & chr5 & 15989524 & 15991470 & 5.65 & 5.83 & 5.96 & 3.85 & 3.72 & 3.86 \\
n337555 & 0.00009 & 9.08 & chrY & 15470990 & 15471751 & 6.51 & 6.76 & 7.06 & 3.78 & 3.23 & 3.78 \\
n334787 & 0.00009 & 31.1 & chr4 & 74608856 & 74609068 & 6.47 & 5.81 & 7.38 & 1.28 & 1.52 & 1.61 \\
\hline
\end{tabular}

Table 3. Top 20 significantly downregulated IncRNAs in HPMECs treated with LPS. Fold: fold change. Chr: chromosome number. LPS 1 to 3 and Ctrl 1 to 3: normalized gene signals of each sample

\begin{tabular}{lccccccccccc}
\hline Accession & P value & Fold & Chr & Gene start & Gene end & LPS & LPS & LPS & Ctrl & Ctrl2 & Ctrl3 \\
\hline NR_001564 & 0.00002 & - & chrX & 73040486 & 73072588 & 3.24 & 3.20 & 3.00 & 8.71 & 8.72 & 8.90 \\
ENST000004244 & 0.00006 & -3.98 & chrX & 3809479 & 3838787 & 5.32 & 5.44 & 5.51 & 7.42 & 7.31 & 7.53 \\
ENST000003811 & 0.00007 & -4.61 & chrX & 3847910 & 3855896 & 5.00 & 4.65 & 4.70 & 7.02 & 6.99 & 6.95 \\
XLOC_010145 & 0.00007 & -4.04 & chr1 & 88775675 & 88813029 & 3.57 & 3.66 & 3.46 & 5.71 & 5.49 & 5.52 \\
ENST000003811 & 0.00009 & -3.50 & chrX & 3771051 & 3800358 & 5.68 & 5.78 & 5.82 & 7.54 & 7.48 & 7.70 \\
n340852 & 0.00010 & -2.53 & chr6 & 13358067 & 13361194 & 6.06 & 6.05 & 5.99 & 7.36 & 7.39 & 7.39 \\
XLOC_12_015669 & 0.00014 & -3.35 & chrX & 3820107 & 3855883 & 5.47 & 5.36 & 5.48 & 7.18 & 7.02 & 7.35 \\
NR_039968 & 0.00018 & -2.46 & chr5 & 72174418 & 72174490 & 4.09 & 4.19 & 4.04 & 5.30 & 5.46 & 5.46 \\
XLOC_008015 & 0.00023 & -2.93 & chrX & 73070670 & 73072528 & 3.49 & 3.58 & 4.02 & 5.28 & 5.25 & 5.20 \\
NR_034031 & 0.00025 & -2.18 & chrX & 3734598 & 3761935 & 5.48 & 5.72 & 5.68 & 6.71 & 6.67 & 6.86 \\
n335593 & 0.00030 & -2.12 & chr1 & 20944648 & 20945246 & 8.87 & 8.92 & 8.98 & 9.83 & 10.0 & 10.1 \\
XL0C_12_006085 & 0.00033 & -2.38 & chr1 & 41426870 & 41428223 & 4.35 & 4.07 & 4.11 & 5.20 & 5.49 & 5.60 \\
XLOC_12_015668 & 0.00040 & -2.34 & chrX & 3782439 & 3799884 & 5.49 & 5.46 & 5.68 & 6.59 & 6.64 & 7.08 \\
n342704 & 0.00053 & -2.87 & chr1 & 41437409 & 41438729 & 4.41 & 4.09 & 4.08 & 5.44 & 5.45 & 6.24 \\
n337065 & 0.00057 & -2.58 & chr5 & 69372740 & 69373356 & 4.81 & 5.22 & 5.55 & 6.39 & 6.44 & 6.84 \\
n334398 & 0.00064 & -2.02 & chr1 & 59479134 & 59480524 & 5.55 & 5.79 & 5.92 & 6.78 & 6.99 & 6.54 \\
NR_037504 & 0.00066 & -2.07 & chr6 & 16741129 & 16741140 & 4.73 & 4.41 & 4.24 & 5.29 & 5.63 & 5.59 \\
XL0C_002531 & 0.00073 & -2.04 & chr2 & 23833756 & 23834346 & 3.84 & 3.61 & 4.23 & 5.01 & 4.82 & 4.92 \\
n336284 & 0.00074 & -2.20 & chr1 & 82761080 & 82761299 & 3.14 & 3.71 & 3.30 & 4.31 & 4.82 & 4.43 \\
ENST000005072 & 0.00075 & -2.06 & chr5 & 33424131 & 33440725 & 4.32 & 3.76 & 4.33 & 5.06 & 5.27 & 5.20 \\
\hline
\end{tabular}

and 29 downregulated mRNAs (Fig. 1B, Fig. 1D). The top 20 most significantly upregulated (Table 4) and downregulated (Table 5) mRNAs are listed below.

Although differentially expressed lncRNAs and mRNAs were widely scattered among all chromosomes, the distribution was not equal (Fig. 1E, Fig. 1F). Chromosome 1 and chromosome 6 had the largest number of differentially expressed mRNAs and IncRNAs, respectively. Chromosome $\mathrm{X}$ had the largest number of downregulated lncRNAs. Fifty-two differentially expressed lncRNAs could not be assigned to corresponding chromosomes. 
Table 4. Top 20 significantly upregulated mRNAs in HPMECs treated with LPS. Fold: fold change. Chr: chromosome number. LPS 1 to 3 and Ctrl 1 to 3: normalized gene signals of each sample

\begin{tabular}{lcccccccccc}
\hline Accession number & Gene symbol & P value & Fold & Chr & LPS1 & LPS2 & LPS3 & Ctrl1 & Ctrl2 & Ctrl3 \\
\hline NM_000450 & SELE & 0.000051 & 86.46 & chr1 & 10.77 & 10.79 & 10.97 & 4.37 & 4.13 & 4.72 \\
NM_000584 & IL8 & 0.000056 & 38.87 & chr4 & 8.87 & 8.83 & 9.20 & 3.93 & 3.52 & 3.61 \\
NM_173054 & RELN & 0.000061 & 19.37 & chr7 & 9.97 & 9.97 & 10.16 & 5.88 & 5.55 & 5.83 \\
NM_001078 & VCAM1 & 0.000066 & 19.21 & chr1 & 7.79 & 7.95 & 8.11 & 3.73 & 3.53 & 3.81 \\
NM_006398 & UBD & 0.000071 & 17.98 & chr6 & 8.08 & 7.86 & 7.95 & 3.62 & 3.86 & 3.90 \\
NM_001511 & CXCL1 & 0.000075 & 16.19 & chr4 & 9.14 & 9.30 & 9.44 & 5.34 & 5.27 & 5.22 \\
NM_177531 & PKHD1L1 & 0.00008 & 16.40 & chr8 & 7.12 & 7.34 & 7.57 & 3.26 & 3.27 & 3.40 \\
NM_001130046 & CCL20 & 0.000085 & 18.04 & chr2 & 6.57 & 6.94 & 7.01 & 2.78 & 2.47 & 2.75 \\
NM_001775 & CD38 & 0.00009 & 15.38 & chr4 & 7.71 & 7.90 & 8.18 & 3.97 & 4.02 & 3.97 \\
NM_001565 & CXCL10 & 0.000095 & 18.10 & chr4 & 6.94 & 7.18 & 7.05 & 2.71 & 2.68 & 3.25 \\
NM_001145938 & MMP1 & 0.000114 & 17.17 & chr11 & 10.53 & 10.56 & 10.66 & 6.79 & 6.09 & 6.56 \\
NM_001128304 & PLSCR4 & 0.000129 & 10.48 & chr3 & 9.99 & 10.04 & 10.29 & 6.85 & 6.53 & 6.77 \\
NM_000072 & CD36 & 0.000134 & 7.82 & chr7 & 5.58 & 5.84 & 5.93 & 2.84 & 2.77 & 2.83 \\
NM_000201 & ICAM1 & 0.000139 & 7.71 & chr19 & 10.53 & 10.53 & 10.56 & 7.83 & 7.48 & 7.47 \\
NM_001008 & RPS4Y1 & 0.000148 & 7.20 & chrY & 6.59 & 6.92 & 6.87 & 3.88 & 3.98 & 3.96 \\
NM_001006624 & PDPN & 0.000153 & 6.74 & chr1 & 8.51 & 8.50 & 8.56 & 5.76 & 5.93 & 5.62 \\
NM_014398 & LAMP3 & 0.000158 & 5.78 & chr3 & 7.90 & 7.91 & 8.09 & 5.47 & 5.40 & 5.44 \\
NM_001781 & CD69 & 0.000163 & 6.69 & chr12 & 5.54 & 5.66 & 5.75 & 3.02 & 2.68 & 3.02 \\
NM_001122665 & DDX3Y & 0.000168 & 6.86 & chrY & 6.25 & 6.39 & 6.75 & 3.67 & 3.61 & 3.77 \\
NM_001712 & CEACAM1 & 0.000173 & 5.97 & chr19 & 8.24 & 8.21 & 8.23 & 5.58 & 5.88 & 5.48 \\
\hline
\end{tabular}

Table 5. Top 20 significantly downregulated mRNAs in HPMECs treated with LPS. Fold: fold change. Chr: chromosome number. LPS 1 to 3 and Ctrl 1 to 3: normalized gene signals of each sample

\begin{tabular}{lcccccccccc}
\hline Accession number & Gene symbol & P value & Fold & Chr & LPS1 & LPS2 & LPS3 & Ctrl1 & Ctrl2 & Ctrl3 \\
\hline NM_001008540 & CXCR4 & 0.000246 & -4.21 & chr2 & 7.27 & 7.16 & 7.20 & 9.30 & 9.09 & 9.46 \\
NM_001130140 & ERAP2 & 0.00046 & -3.26 & chr5 & 4.97 & 5.25 & 5.24 & 6.72 & 6.81 & 7.04 \\
NM_018659 & CYTL1 & 0.000528 & -3.17 & chr4 & 8.24 & 8.18 & 8.11 & 9.57 & 9.90 & 10.05 \\
NM_019035 & PCDH18 & 0.000586 & -2.72 & chr4 & 5.54 & 5.58 & 5.46 & 6.84 & 7.11 & 6.96 \\
BC056414 & PLVAP & 0.000601 & -2.58 & chr19 & 6.94 & 6.84 & 6.89 & 8.18 & 8.39 & 8.19 \\
NM_004476 & FOLH1 & 0.000703 & -2.37 & chr11 & 4.45 & 4.31 & 4.42 & 5.61 & 5.55 & 5.76 \\
NM_153696 & FOLH1B & 0.000708 & -2.48 & chr11 & 3.56 & 3.72 & 3.68 & 4.99 & 4.76 & 5.14 \\
NM_006366 & CAP2 & 0.000722 & -2.31 & chr6 & 5.75 & 5.64 & 5.87 & 6.97 & 6.91 & 7.00 \\
NM_021194 & SLC30A1 & 0.00079 & -3.13 & chr1 & 6.01 & 5.90 & 6.09 & 6.95 & 7.96 & 8.02 \\
NM_001198 & PRDM1 & 0.000839 & -2.45 & chr6 & 5.52 & 5.58 & 5.58 & 7.10 & 6.50 & 6.95 \\
NM_001242628 & GFOD1 & 0.000844 & -2.08 & chr6 & 6.19 & 6.16 & 6.09 & 7.15 & 7.25 & 7.20 \\
NM_001199989 & RASD1 & 0.000854 & -2.21 & chr17 & 4.92 & 4.85 & 4.93 & 5.85 & 6.09 & 6.19 \\
NM_003654 & CHST1 & 0.000888 & -2.04 & chr11 & 7.19 & 7.23 & 7.12 & 8.15 & 8.22 & 8.24 \\
NM_001114403 & UPK3BL & 0.000897 & -2.10 & chr7 & 8.10 & 8.03 & 7.94 & 9.04 & 9.04 & 9.19 \\
NM_006208 & ENPP1 & 0.001009 & -2.20 & chr6 & 4.39 & 4.54 & 4.51 & 5.37 & 5.55 & 5.93 \\
NM_004701 & CCNB2 & 0.001019 & -2.32 & chr15 & 5.47 & 5.63 & 5.66 & 6.39 & 6.88 & 7.14 \\
NM_021958 & HLX & 0.001063 & -2.02 & chr1 & 6.73 & 6.67 & 6.47 & 7.67 & 7.74 & 7.52 \\
NM_003525 & HIST1H2BI & 0.001072 & -2.41 & chr6 & 5.44 & 5.33 & 5.52 & 6.26 & 6.64 & 7.19 \\
NM_003537 & HIST1H3B & 0.001077 & -2.46 & chr6 & 5.79 & 5.75 & 5.83 & 6.58 & 7.06 & 7.61 \\
NM_004460 & FAP & 0.001131 & -2.01 & chr2 & 3.88 & 3.85 & 4.04 & 4.88 & 4.79 & 5.12 \\
\hline
\end{tabular}

\section{GO and KEGG enrichment analyses}

To explore the role of differentially expressed mRNAs in HPMECs treated with LPS, we performed GO and KEGG pathway enrichment analysis.

The results showed that upregulated genes were mainly associated with the following functions: response to lipopolysaccharide (ontology: biological process), integrin complex (ontology: cellular component), and cytokine activity (ontology: molecular function) (Fig. 2A). Downregulated genes were mainly associated with the following functions: male pronucleus (ontology: cellular component), C-terminal protein deglutamylation (ontology: biological process), and exopeptidase activity (ontology: molecular function) (Fig. 2B). The 




Fig. 2. GO and KEGG enrichment analyses for upregulated and downregulated mRNAs. GO enrichment analysis of upregulated (A) and downregulated (B) genes (Top 10, p<0.05). KEGG enrichment analysis for upregulated (C) and downregulated (D) genes $(\mathrm{p}<0.05)$. The red to green colors indicate high to low $-\log (\mathrm{p}$ value) levels. Point size indicates the number of differentially expressed genes in the corresponding pathway.

results also indicated that upregulated genes were mainly associated with the following pathways: TNF signaling pathway, cytokine-cytokine receptor interaction, rheumatoid arthritis, NOD-like receptor signaling pathway, and cell adhesion molecules (CAMs) (Fig. 2C). The five most enriched pathways of downregulated genes were mineral absorption, progesterone-mediated oocyte maturation, riboflavin metabolism, cell cycle, and systemic lupus erythematosus (Fig. 2D).

These data suggested that upregulated mRNAs may directly participate in the process of pulmonary endothelial inflammation and barrier dysfunction.

\section{LncRNA-mRNA coexpression networks with GO and KEGG enrichment analysis}

To explore the potential biological functions of IncRNAs in HPMECs treated with LPS, we constructed a lncRNA-mRNA coexpression network based on 72 differentially expressed lncRNAs and 132 interacting differentially expressed mRNAs (Fig. 3A). Then, we performed GO and KEGG pathway enrichment analysis on the 132 mRNAs. We found that the most 


\section{Cellular Physiology Cell Physiol Biochem 2019;52:653-667 \begin{tabular}{ll|l} 
and Biochemistry & $\begin{array}{l}\text { DOl: 10.33594/000000046 } \\
\text { Published online: 29 March 2019 }\end{array}$ & $\begin{array}{l}\text { O 2019 The Author(s). Published by } \\
\text { Cell Physiol Biochem Press GmbH\&Co. KG }\end{array}$ \\
\cline { 2 - 3 }
\end{tabular}

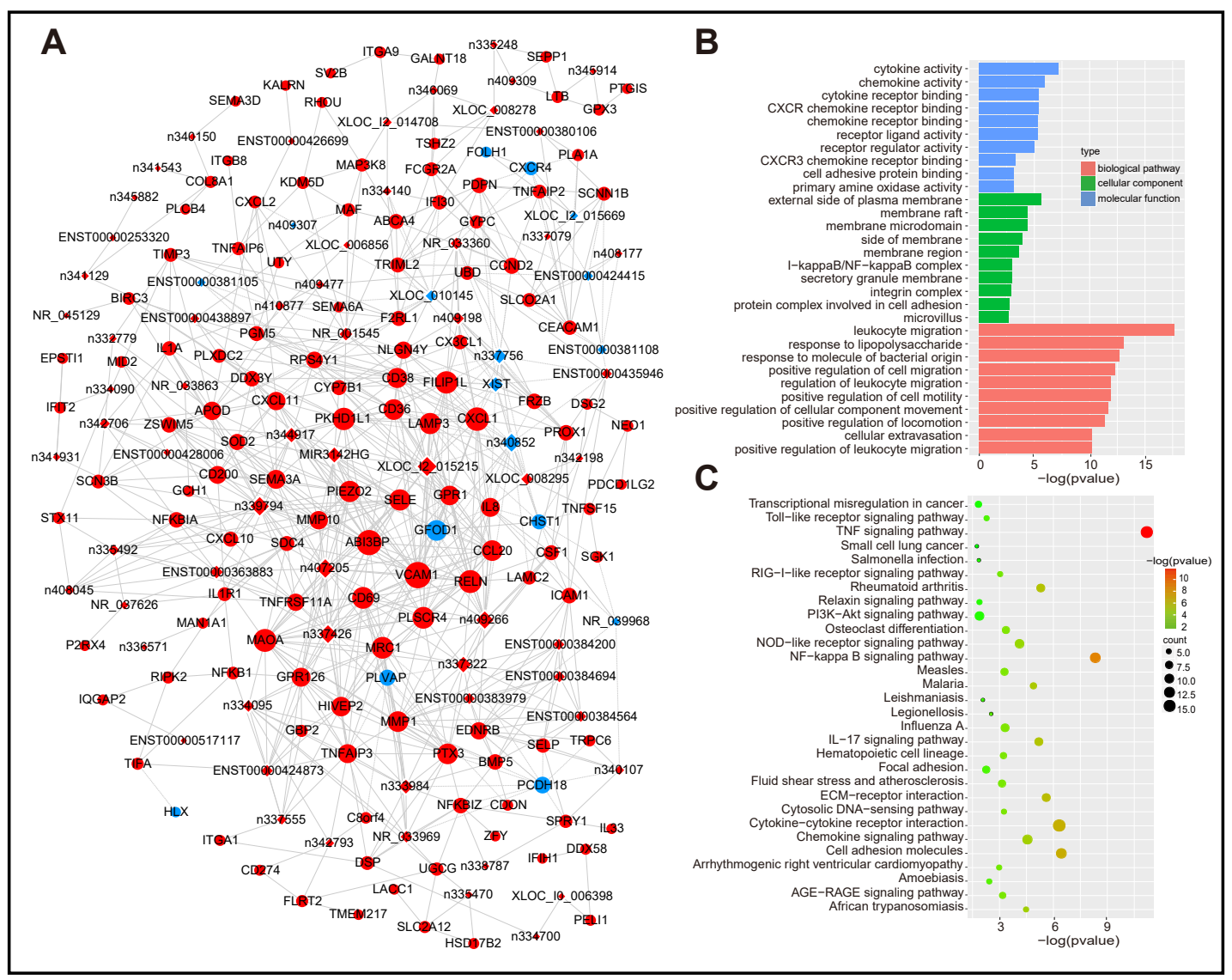

Fig. 3. Coexpression network of 72 differentially expressed lncRNAs and 132 interacting differentially expressed mRNAs with GO and KEGG analysis results. Coexpression network (A) of 72 lncRNAs and 132 interacting mRNAs. The diamonds represent lncRNAs, and the circles represent their correlated mRNAs. Blue dots and red dots indicate downregulated and upregulated IncRNAs and mRNAs. GO enrichment analysis (B) and KEGG enrichment analysis (C) of the 132 differentially expressed mRNAs ( $\mathrm{p}<0.05)$.

enriched GOs were leukocyte migration (ontology: biological process), cytokine activity (ontology: molecular function), and external side of plasma membrane (ontology: cellular component) (Fig. 3B). The results also indicated that the $132 \mathrm{mRNAs}$ were mainly associated with the following pathways: TNF signaling pathway, NF- $\kappa B$ signaling pathway, CAMs, cytokine-cytokine receptor interaction, and ECM-receptor interaction (Fig. 3C).

\section{Real-time PCR validation of the microarray data}

To validate the reliability of the microarray analysis results and to provide a research basis for further study, the expression levels of 6 lncRNAs (MIR3142HG, n344917, XLOC_ 12_015215, n340107, n407205 and XIST) and 8 mRNAs (SELE, IL8, VCAM1, ICAM1, CXCL10, MMP10, ABI3BP and TRPC6) were determined by real-time PCR (Fig. 4). The results showed that MIR3142HG, XLOC_12_015215, n340107 and n407205 were upregulated, whereas XIST was significantly downregulated. The expression of n344917 showed no significant change. SELE, IL8, VCAM1, ICAM1, CXCL10, MMP10, ABI3BP and TRPC6 were upregulated.

These data suggest that the expression levels of selected lncRNAs and mRNAs, except for n344917, detected by real-time PCR were similar to those detected by microarray analysis. 
Cellular Physiology Cell Physiol Biochem 2019;52:653-667

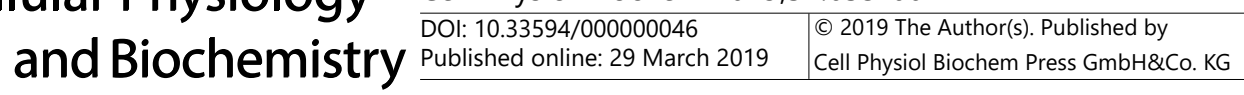

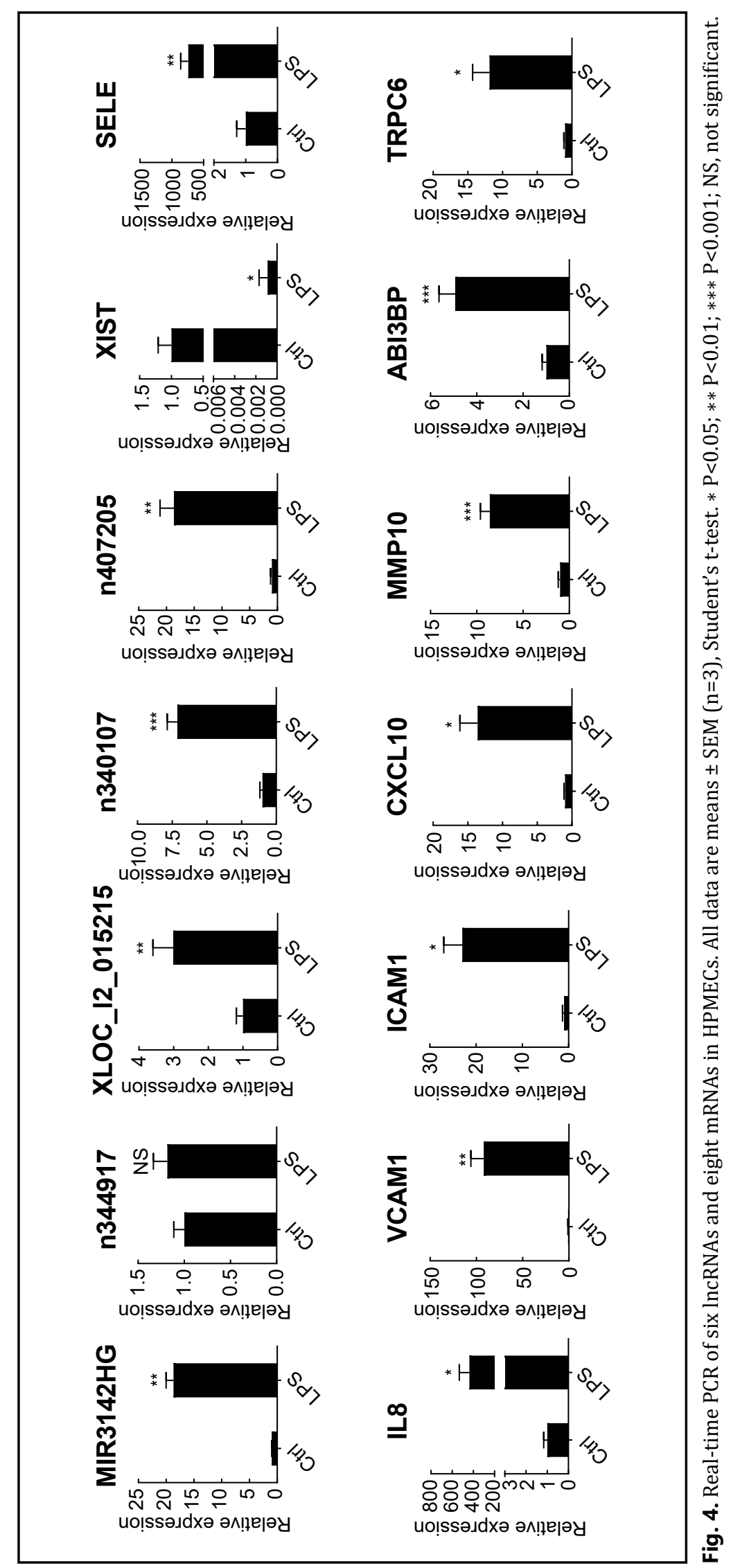




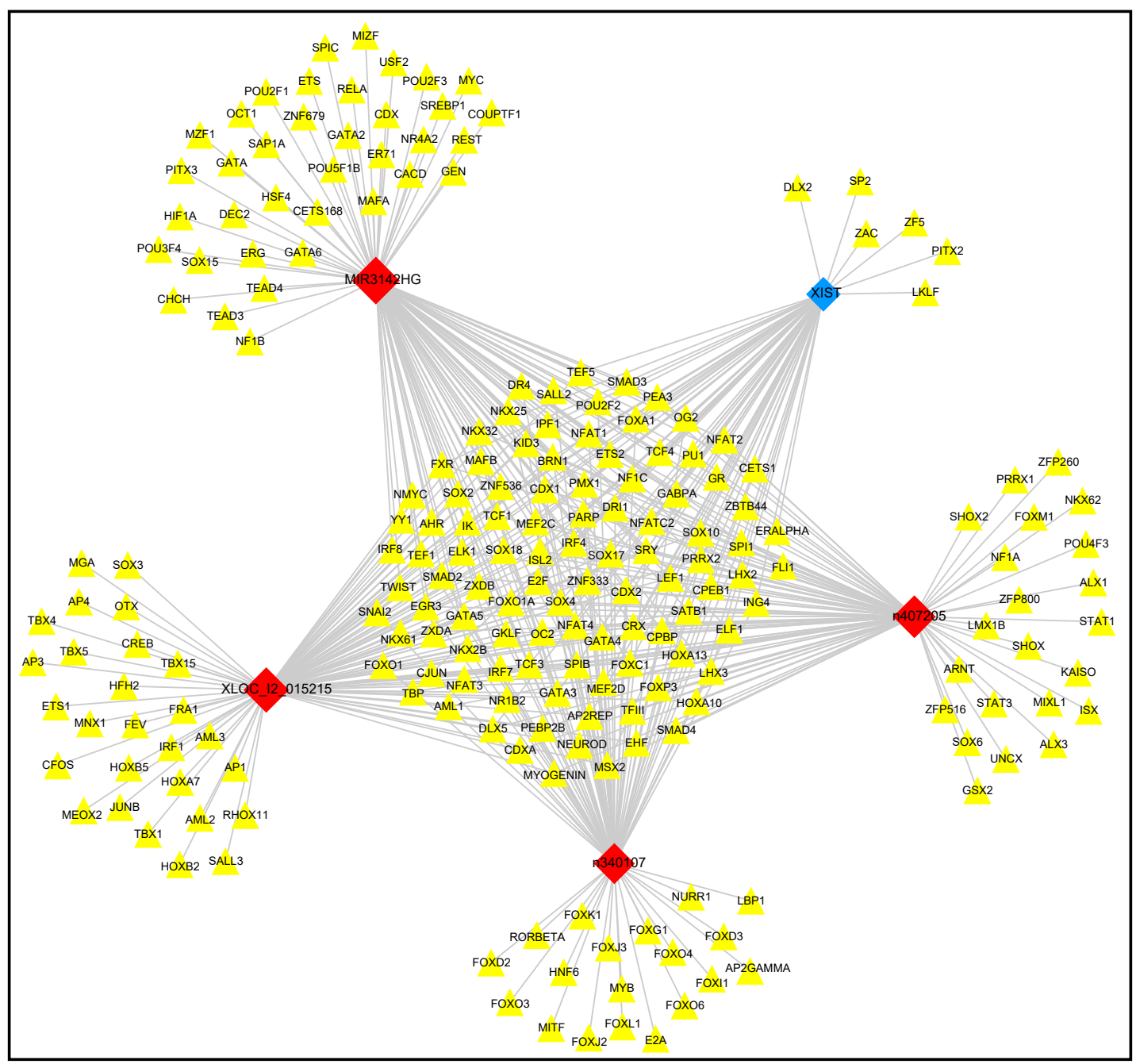

Fig. 5. TF regulatory network of the five validated lncRNAs. The diamonds represent lncRNAs. Blue and red dots indicate downregulated and upregulated lncRNAs. Yellowish triangles represent correlated TFs.

\section{TF-lncRNA regulatory network}

To understand the reason for the differential expression of the five validated lncRNAs (MIR3142HG, XLOC_12_015215, n340107, n407205 and XIST), we predicted TFs mapping to these IncRNAs in the TRANSFACT professional database. The results showed that of the 214 TFs mapping to these IncRNAs (Fig. 5), 17 out of the 214 TFs were differently expressed (fold change $>1.2, p<0.05$ ). Seven differentially expressed TFs were predicted to regulate the transcription of XIST (Table 6), including 2 upregulated TFs (POU2F2 and BCL6) and 5 downregulated mRNAs (SOX18, MEF2C, SOX17, HOXB5 and ETS2). These findings indicate that differently expressed TFs might be one of the causes of the differential expression of lncRNAs.

\section{Discussion}

In this study, we established an experimental model of pulmonary endothelial inflammation and barrier dysfunction by stimulating HPMECs with LPS and investigated the expression profile of IncRNAs and mRNAs by microarray analysis. The results indicate that IncRNAs play an important role in LPS-induced pulmonary endothelial inflammation and barrier dysfunction. 
Table 6. Differentially expressed TFs predicted to regulate the transcription of XIST. Range: the promoter region of XIST. Position: the position of the transcription start site. Sequence: the TF binding sequences in the promoter region

\begin{tabular}{|c|c|c|c|c|c|}
\hline IncRNA & Range & Transcription factor & Relation & Position & sequence \\
\hline XIST & chrX :-:73852253-73854253 & S0X18 & down & 690 & CAATGgg \\
\hline XIST & chrX :-:73852253-73854253 & S0X18 & down & 724 & gaCATTG \\
\hline XIST & chrX :-:73852253-73854253 & S0X18 & down & 1536 & CAATGgc \\
\hline XIST & chrX :-:73852253-73854253 & MEF2C & down & 8 & aаAAATA \\
\hline XIST & chrX :-:73852253-73854253 & MEF2C & down & 94 & aaAAATA \\
\hline XIST & chrX :-:73852253-73854253 & MEF2C & down & 265 & aaAAATA \\
\hline XIST & chrX :-:73852253-73854253 & MEF2C & down & 317 & aaAAATA \\
\hline XIST & chrX :-:73852253-73854253 & MEF2C & down & 1665 & aaAAATA \\
\hline XIST & chrX :-:73852253-73854253 & SoX17 & down & 597 & gGACAA \\
\hline XIST & chrX :-:73852253-73854253 & HOXB5 & down & 743 & aatAATTAat \\
\hline XIST & chrX :-:73852253-73854253 & ETS2 & down & 428 & caGGAAG \\
\hline XIST & chrX :-:73852253-73854253 & POU2F2 & up & 1678 & TATGCaaat \\
\hline XIST & chrX :-:73852253-73854253 & BCL6 & up & 391 & ttctAGAAAg \\
\hline XIST & chrX :-:73852253-73854253 & BCL6 & up & 1565 & ttctAGAAAg \\
\hline
\end{tabular}

An overwhelming majority of the transcriptions of the human genome are ncRNAs, which act as important transcriptional regulators in pathophysiologic processes [31-33]. In contrast to miRNAs, which regulate target genes by a posttranscriptional mechanism $[34,35]$, IncRNAs have the potential to regulate gene expression at posttranscriptional, transcriptional and epigenetic levels [36-39]. Furthermore, many lncRNAs have shown developmental stage-specific and tissue-specific expression patterns [40, 41].

The roles of ncRNAs in the pathogenesis of ALI and ARDS have attracted increasing attention. Recent studies have demonstrated that several miRNAs, such as miR-146, miR155, and miR-221, serve as important regulators of inflammation-related mediators [33]. Hongbin Li et al. reported that the IncRNA CASC2 improved ALI by reducing lung epithelial cell apoptosis [42]. However, the function of IncRNA has not been investigated in pulmonary vascular injury associated with ALI and ARDS. Unlike two previous studies that analyzed the lncRNA expression profile in human umbilical vein endothelial cells [43] and human dermal microvascular endothelial cells [44] exposed to LPS, we identified the expression of LPSresponsive IncRNAs in HPMECs to investigate the role of IncRNAs in pulmonary endothelial injury.

In the present study, we determined the expression of 6 lncRNAs and 8 mRNAs by realtime PCR. The selection of these lncRNAs and mRNAs was based on the fold change and degree data in the IncRNA-mRNA coexpression network. Our results showed that the lncRNA and mRNA expression results determined by microarray analysis had good reliability and reproducibility. LPS successfully activated HPMECs because the expression of SELE, IL-8, VCAM-1, CCL20 and ICAM-1 increased significantly in the experimental model [45-47]. In addition, GO and KEGG pathway enrichment analyses also suggested that the differentially expressed mRNAs participated in pulmonary endothelial inflammation and barrier dysfunction. Notably, based on GO and KEGG pathway enrichment analyses of the lncRNAmRNA coexpression network, we found that 72 differentially expressed lncRNAs might be mainly involved in the TNF signaling pathway, NF- $\kappa$ B signaling pathway, CAMs, cytokinecytokine receptor interactions, and ECM-receptor interactions $[48,49]$. Thus, we speculated that these IncRNAs might be associated with pulmonary endothelial inflammation and barrier dysfunction. Furthermore, we predicted differentially expressed TFs mapping to five selected lncRNAs that had been verified by real-time PCR with the help of the TRANSFAC professional database. The results suggested that differently expressed TFs might be one of the causes of the differential expression of lncRNAs. 


\section{Cellular Physiology Cell Physiol Biochem 2019;52:653-667 \\ \begin{tabular}{c|c|c|c|}
\hline DOI: 10.33594/000000046 & 2019 The Author(s). Published by \\
\hline and Biochemistry
\end{tabular} \\ \begin{tabular}{ll} 
Published online: 29 March 2019 Cell Physiol Biochem Press GmbH\&Co. KG \\
\hline Wh
\end{tabular} \\ Wang et al.: IncRNA in HPMECs Exposed to LPS}

The understanding of the diversity of gene regulation has greatly expanded in the past decade. There is increasing recognition that ncRNAs are important components of the gene regulatory network [50]. As the roles of IncRNAs become clearer, the knowledge acquired by this research will enable the understanding of how lncRNAs affect the initiation, progression, and resolution of pulmonary endothelial dysfunction associated with ALI and ARDS. Our data still need to be further validated in both vitro and vivo.

$\mathrm{XIST}$, which is the master regulator of $\mathrm{X}$ chromosome inactivation, has been reported to play an important role in the pathogenesis of many diseases [51, 52]. $\mathrm{H} \mathrm{Yu}$ et al. reported that XIST inhibition increased the blood-tumor barrier permeability in glioma endothelial cells [53]. Here, we found that XIST was significantly downregulated in HPMECs exposed to LPS by both microarray data and real-time PCR. This is the first study to determine the expression of XIST in HPMECs. The results may be helpful for further insights into the underlying role and mechanism of XIST in pulmonary endothelial inflammation and barrier dysfunction. The detailed function of XIST in pulmonary endothelial injury still needs to be further investigated.

Although not a genetic disease, ARDS has a certain hereditary susceptibility [54]. Three retrospective studies reported that men were at higher risk than women of incidence and mortality from ARDS [55-57]. In this study, we found that the distribution of differentially expressed lncRNAs was not equal. Interestingly, most differentially expressed lncRNAs on chromosome $\mathrm{X}$ were downregulated, while all differentially expressed IncRNAs on chromosome $Y$ were upregulated. We speculate that the differential expression of IncRNAs on chromosomes $\mathrm{X}$ and $\mathrm{Y}$ may be one of the causes of sex-related differences in the morbidity and mortality of ARDS. Of course, this subject still requires more clinical research and further investigations.

\section{Conclusion}

In conclusion, we investigated the expression of IncRNAs and mRNAs in HPMECs treated with LPS by microarray analysis. We found that IncRNAs may be involved in LPS-induced pulmonary endothelial inflammation and barrier dysfunction. This is the first study to reveal the expression profile and potential role of lncRNAs in HPMECs. These findings provide a novel direction for both basic and clinical research for ALI and ARDS. Due to opportunities to identify novel therapeutic and preventive targets, our results may provide relevant information for future clinical interventions of ALI and ARDS.

\section{Acknowledgements}

This work was supported by the National Natural Science Foundation of China (No. 81770076, No. 81570074 and No. 81600054) and Joint Project of Shandong Natural Science Foundation (ZR2015HL002).

\section{Disclosure Statement}

The authors have no conflicts of interest to declare. 


\section{Cellular Physiology Cell Physiol Biochem 2019;52:653-667 \begin{tabular}{ll|l}
\cline { 2 - 3 } DOI: 10.33594/000000046 & (c) 2019 The Author(s). Published by
\end{tabular} and Biochemistry Published online: 29 March $2019 \quad$ Cell Physiol Biochem Press GmbH\&Co. KG \\ Wang et al.: IncRNA in HPMECs Exposed to LPS}

\section{References}

- 1 Matthay MA, Zemans RL: The acute respiratory distress syndrome: pathogenesis and treatment. Annu Rev Pathol 2011;6:147-163.

- 2 Matthay MA, Ware LB, Zimmerman GA: The acute respiratory distress syndrome. J Clin Invest 2012;122:2731-2740.

- 3 Lee KY: Pneumonia, Acute Respiratory Distress Syndrome, and Early Immune-Modulator Therapy. Int J Mol Sci 2017;18:pii:E388.

- 4 Yang CY, Chen CS, Yiang GT, Cheng YL, Yong SB, Wu MY, Li CJ: New Insights into the Immune Molecular Regulation of the Pathogenesis of Acute Respiratory Distress Syndrome. Int J Mol Sci 2018;19:pii:E588.

5 Fan E, Brodie D, Slutsky AS: Acute Respiratory Distress Syndrome: Advances in Diagnosis and Treatment. JAMA 2018;319:698-710.

- 6 Wohlrab P, Kraft F, Tretter V, Ullrich R, Markstaller K, Klein KU: Recent advances in understanding acute respiratory distress syndrome. F1000Res 2018; DOI:10.12688/f1000research.

7 Quinn JJ, Chang HY: Unique features of long non-coding RNA biogenesis and function. Nat Rev Genet 2016;17:47-62.

8 Batista PJ, Chang HY: Long noncoding RNAs: Cellular address codes in development and disease. Cell 2013;152:1298-1307.

- 9 Rinn JL, Chang HY: Genome regulation by long noncoding RNAs. Annu Rev Biochem 2012;81:145-166.

- 10 Ponting CP, Oliver PL, Reik W: Evolution and functions of long noncoding RNAs. Cell 2009;136:629-641.

- 11 Tao Z, Yuan Y, Liao Q: Alleviation of Lipopolysaccharides-Induced Acute Lung Injury by miR-454. Cell Physiol Biochem 2016;38:65-74.

12 Cai ZG, Zhang SM, Zhang Y, Zhou YY, Wu HB, Xu XP: MicroRNAs are dynamically regulated and play an important role in LPS-induced lung injury. Can J Physiol Pharmacol 2012;90:37-43.

- 13 Li W, Qiu X, Jiang H, Han Y, Wei D, Liu J: Downregulation of miR-181a protects mice from LPS-induced acute lung injury by targeting Bcl-2. Biomed Pharmacother 2016;84:1375-1382.

- 14 Fang Y, Gao F, Hao J, Liu Z: microRNA-1246 mediates lipopolysaccharide-induced pulmonary endothelial cell apoptosis and acute lung injury by targeting angiotensin-converting enzyme 2 . Am J Transl Res 2017;9:1287-1296.

15 Xie T, Liang J, Liu N, Wang Q, Li Y, Noble PW, Jiang D: MicroRNA-127 inhibits lung inflammation by targeting IgG Fcgamma receptor I. J Immunol 2012;188:2437-2444.

16 Ying H, Kang Y, Zhang H, Zhao D, Xia J, Lu Z, Wang H, Xu F, Shi L: MiR-127 modulates macrophage polarization and promotes lung inflammation and injury by activating the JNK pathway. J Immunol 2015;194:1239-1251.

- 17 Davidson-Moncada J, Papavasiliou FN, Tam W: MicroRNAs of the immune system: roles in inflammation and cancer. Ann N Y Acad Sci 2010;1183:183-194.

- 18 Vergadi E, Vaporidi K, Theodorakis EE, Doxaki C, Lagoudaki E, Ieronymaki E, Alexaki VI, Helms M, Kondili E, Soennichsen B, Stathopoulos EN, Margioris AN, Georgopoulos D, Tsatsanis C: Akt2 deficiency protects from acute lung injury via alternative macrophage activation and miR-146a induction in mice. J Immunol 2014;192:394-406.

- 19 Zeng Z, Gong H, Li Y, Jie K, Ding C, Shao Q Liu F, Zhan Y, Nie C, Zhu W, Qian K: Upregulation of miR-146a contributes to the suppression of inflammatory responses in LPS-induced acute lung injury. Exp Lung Res 2013;39:275-282.

20 Han Y, Li Y, Jiang Y: The Prognostic Value of Plasma MicroRNA-155 and MicroRNA-146a Level in Severe Sepsis and Sepsis-Induced Acute Lung Injury Patients. Clin Lab 2016;62:2355-2360.

21 Li H, He B, Liu X, Li J, Liu Q Dong W, Xu Z, Qian G, Zuo H, Hu C, Qian H, Mao C, Wang G: Regulation on Toll-like Receptor 4 and Cell Barrier Function by Rab26 siRNA-loaded DNA Nanovector in Pulmonary Microvascular Endothelial Cells. Theranostics 2017;7:2537-2554.

22 Nickols J, Obiako B, Ramila KC, Putinta K, Schilling S, Sayner SL: Lipopolysaccharide-induced pulmonary endothelial barrier disruption and lung edema: critical role for bicarbonate stimulation of AC10. Am J Physiol Lung Cell Mol Physiol 2015;309:L1430-1437.

- 23 Menden H, Tate E, Hogg N, Sampath V: LPS-mediated endothelial activation in pulmonary endothelial cells: role of Nox2-dependent IKK-beta phosphorylation. Am J Physiol Lung Cell Mol Physiol 2013;304:L445-455. 


\section{Cellular Physiology Cell Physiol Biochem 2019;52:653-667 \begin{tabular}{ll|l|l} 
DOI: 10.33594/000000046 & (O) 2019 The Author(s). Published by \\
\hline
\end{tabular} and Biochemistry Published online: 29 March 2019 Cell Physiol Biochem Press GmbH\&Co. KG \\ Wang et al.: IncRNA in HPMECs Exposed to LPS}

24 Li L, Hu J, He T, Zhang Q Yang X, Lan X, Zhang D, Mei H, Chen B, Huang Y: P38/MAPK contributes to endothelial barrier dysfunction via MAP4 phosphorylation-dependent microtubule disassembly in inflammation-induced acute lung injury. Sci Rep 2015;5:8895.

- 25 Dreymueller D, Martin C, Kogel T, Pruessmeyer J, Hess FM, Horiuchi K, Uhlig S, Ludwig A: Lung endothelial ADAM17 regulates the acute inflammatory response to lipopolysaccharide. EMBO Mol Med 2012;4:412423.

- 26 Irizarry RA, Bolstad BM, Collin F, Cope LM, Hobbs B, Speed TP: Summaries of Affymetrix GeneChip probe level data. Nucleic Acids Res 2003;31:e15.

27 Smyth GK: Linear models and empirical bayes methods for assessing differential expression in microarray experiments. Stat Appl Genet Mol Biol 2004;3:Article3.

28 Livak KJ, Schmittgen TD: Analysis of relative gene expression data using real-time quantitative PCR and the 2(-Delta Delta C(T)) Method. Methods 2001;25:402-408.

29 Pujana MA, Han JD, Starita LM, Stevens KN, Tewari M, Ahn JS, Rennert G, Moreno V, Kirchhoff T, Gold B, Assmann V, Elshamy WM, Rual JF, Levine D, Rozek LS, Gelman RS, Gunsalus KC, Greenberg RA, Sobhian B, Bertin N, et al.: Network modeling links breast cancer susceptibility and centrosome dysfunction. Nat Genet 2007;39:1338-1349.

- 30 Prieto C, Risueno A, Fontanillo C, De las Rivas J: Human gene coexpression landscape: confident network derived from tissue transcriptomic profiles. PLoS One 2008;3:e3911.

31 Hackermuller J, Reiche K, Otto C, Hosler N, Blumert C, Brocke-Heidrich K, Bohlig L, Nitsche A, Kasack K, Ahnert P, Krupp W, Engeland K, Stadler PF, Horn F: Cell cycle, oncogenic and tumor suppressor pathways regulate numerous long and macro non-protein-coding RNAs. Genome Biol 2014;15:R48.

- 32 Cardinal-Fernandez P, Ferruelo A, Esteban A, Lorente JA: Characteristics of microRNAs and their potential relevance for the diagnosis and therapy of the acute respiratory distress syndrome: from bench to bedside. Transl Res 2016;169:102-111.

- 33 Marques-Rocha JL, Samblas M, Milagro FI, Bressan J, Martinez JA, Marti A: Noncoding RNAs, cytokines, and inflammation-related diseases. FASEB J 2015;29:3595-3611.

34 Rebane A, Akdis CA: MicroRNAs: Essential players in the regulation of inflammation. J Allergy Clin Immunol 2013;132:15-26.

35 Djuranovic S, Nahvi A, Green R: A parsimonious model for gene regulation by miRNAs. Science 2011;331:550-553.

36 Prensner JR, Chinnaiyan AM: The emergence of lncRNAs in cancer biology. Cancer Discov 2011;1:391-407.

- 37 Villegas VE, Zaphiropoulos PG: Neighboring gene regulation by antisense long non-coding RNAs. Int J Mol Sci 2015;16:3251-3266.

- 38 Karapetyan AR, Buiting C, Kuiper RA, Coolen MW: Regulatory Roles for Long ncRNA and mRNA. Cancers (Basel) 2013;5:462-490.

- 39 Angrand PO, Vennin C, Le Bourhis X, Adriaenssens E: The role of long non-coding RNAs in genome formatting and expression. Front Genet 2015;6:165.

40 Jehan Z, Vallinayagam S, Tiwari S, Pradhan S, Singh L, Suresh A, Reddy HM, Ahuja YR, Jesudasan RA: Novel noncoding RNA from human Y distal heterochromatic block (Yq12) generates testis-specific chimeric CDC2L2. Genome Res 2007;17:433-440.

41 Kutter C, Watt S, Stefflova K, Wilson MD, Goncalves A, Ponting CP, Odom DT, Marques AC: Rapid turnover of long noncoding RNAs and the evolution of gene expression. PLoS Genet 2012;8:e1002841.

$42 \mathrm{Li} \mathrm{H}$, Shi H, Gao M, Ma N, Sun R: Long non-coding RNA CASC2 improved acute lung injury by regulating miR-144-3p/AQP1 axis to reduce lung epithelial cell apoptosis. Cell Biosci 2018;8:15.

43 Singh KK, Matkar PN, Muhammad S, Quan A, Gupta V, Teoh H, Al-Omran M, Verma S: Investigation of novel LPS-induced differentially expressed long non-coding RNAs in endothelial cells. Mol Cell Biochem 2016;421:157-168.

- 44 Chowdhury IH, Narra HP, Sahni A, Khanipov K, Schroeder CLC, Patel J, Fofanov Y, Sahni SK: Expression Profiling of Long Noncoding RNA Splice Variants in Human Microvascular Endothelial Cells: Lipopolysaccharide Effects In vitro. Mediators Inflamm 2017;2017:3427461.

- 45 Liu G, Place AT, Chen Z, Brovkovych VM, Vogel SM, Muller WA, Skidgel RA, Malik AB, Minshall RD: ICAM1-activated Src and eNOS signaling increase endothelial cell surface PECAM-1 adhesivity and neutrophil transmigration. Blood 2012;120:1942-1952. 


\section{Cellular Physiology and Biochemistry}

Cell Physiol Biochem 2019;52:653-667

\begin{tabular}{l|l}
\hline DOI: $10.33594 / 000000046$ & (c) 2019 The Author(s). Published by
\end{tabular}

Wang et al.: IncRNA in HPMECs Exposed to LPS

- 46 Pober JS, Sessa WC: Evolving functions of endothelial cells in inflammation. Nat Rev Immunol 2007;7:803815.

- 47 Zhou M, Ding WJ, Chen YW, Shen F, Zeng JY, Qu CY, Wei YF, Xu LM: Expression Changes of Long Noncoding RNA in the Process of Endothelial Cell Activation. Cell Physiol Biochem 2017;41:115-123.

- 48 van der Poll T, van de Veerdonk FL, Scicluna BP, Netea MG: The immunopathology of sepsis and potential therapeutic targets. Nat Rev Immunol 2017;17:407-420.

49 Danese S, Dejana E, Fiocchi C: Immune regulation by microvascular endothelial cells: directing innate and adaptive immunity, coagulation, and inflammation. J Immunol 2007;178:6017-6022.

50 Wang KC, Chang HY: Molecular mechanisms of long noncoding RNAs. Mol Cell 2011;43:904-914.

51 Pintacuda G, Young AN, Cerase A: Function by Structure: Spotlights on Xist Long Non-coding RNA. Front Mol Biosci 2017;4:90.

52 Xiong Y, Wang L, Li Y, Chen M, He W, Qi L: The Long Non-Coding RNA XIST Interacted with MiR-124 to Modulate Bladder Cancer Growth, Invasion and Migration by Targeting Androgen Receptor (AR). Cell Physiol Biochem 2017;43:405-418.

53 Yu H, Xue Y, Wang P, Liu X, Ma J, Zheng J, Li Z, Li Z, Cai H, Liu Y: Knockdown of long non-coding RNA XIST increases blood-tumor barrier permeability and inhibits glioma angiogenesis by targeting miR-137. Oncogenesis 2017;6:e303.

54 Gao L, Barnes KC: Recent advances in genetic predisposition to clinical acute lung injury. Am J Physiol Lung Cell Mol Physiol 2009;296:L713-725.

- 55 Dodoo-Schittko F, Brandstetter S, Brandl M, Blecha S, Quintel M, Weber-Carstens S, Kluge S, Meybohm P, Rolfes C, Ellger B, Bach F, Welte T, Muders T, Thomann-Hackner K, Bein T, Apfelbacher C: Characteristics and provision of care of patients with the acute respiratory distress syndrome: descriptive findings from the DACAPO cohort baseline and comparison with international findings. J Thorac Dis 2017;9:818-830.

56 Dodoo-Schittko F, Brandstetter S, Brandl M, Blecha S, Quintel M, Weber-Carstens S, Kluge S, Kirschning T, Muders T, Bercker S, Ellger B, Arndt C, Meybohm P, Adamzik M, Goldmann A, Karagiannidis C, Bein T, Apfelbacher C, DACAPO Study Group: German-wide prospective DACAPO cohort of survivors of the acute respiratory distress syndrome (ARDS): a cohort profile. BMJ Open 2018;8:e019342.

57 Cochi SE, Kempker JA, Annangi S, Kramer MR, Martin GS: Mortality Trends of Acute Respiratory Distress Syndrome in the United States from 1999 to 2013. Ann Am Thorac Soc 2016;13:1742-1751. 